\title{
Resveratrol exerts growth inhibitory effects on human SZ95 sebocytes through the inactivation of the PI3-K/Akt pathway
}

\author{
SO YOUNG KIM ${ }^{1,2}$, MOO YEOL HYUN ${ }^{2}$, KYUNG CHAN GO ${ }^{1,2}$, \\ CHRISTOS C. ZOUBOULIS ${ }^{3}$ and BEOM JOON KIM ${ }^{1,2}$ \\ ${ }^{1}$ Major in Biomedical Science, Department of Medicine, Chung-Ang University, \\ ${ }^{2}$ Department of Dermatology, Chung-Ang University College of Medicine, Seoul 156-756, Republic of Korea; \\ ${ }^{3}$ Departments of Dermatology, Venereology, Allergology and Immunology, Dessau Medical Center, Dessau 06847, Germany
}

Received November 1, 2014; Accepted January 19, 2015

DOI: 10.3892/ijmm.2015.2098

\begin{abstract}
Resveratrol is known to be a naturally produced polyphenol that is able to reduce cell proliferation in several types of cancer cells and adipocytes. However, the antiproliferative effects of resveratrol on the growth of human sebocytes are not yet clear. In the present study, we investigated possible cellular pathways associated with these growth inhibitory effects on human SZ95 sebocytes. Our results revealed that resveratrol inhibited the proliferation of sebocytes, and that this resulted in disruption of the cell cycle. The inactivation of extracellular signal-regulated protein kinase (ERK), Akt and peroxisome proliferator-activated receptor (PPAR)- $\gamma$ was also shown to be involved in the inhibition of sebocyte growth by resveratrol. To examine the antiproliferative effects of resveratrol, we determined the levels of cell cycle control proteins. Resveratrol inhibited cyclin D1 synthesis, whereas it stimulated $\mathrm{p} 21^{\mathrm{WAF} / \mathrm{CIP1}}$ (p21) and $\mathrm{p} 27^{\mathrm{KIPl}}$ (p27) synthesis. In addition, we demonstrated that the resveratrol-mediated cell cycle arrest resulted in an increase in the proportion of cells in the sub- $\mathrm{G}_{0} / \mathrm{G}_{1}$ phase. Moreover, we found that the growth inhibitory effects of resveratrol were enhanced by treatment with LY294002 [a phosphatidylinositol 3-kinase (PI3-K) inhibitor] more so than by treatment with PD98059 (a MEK inhibitor), which indicates that resveratrol exerts its inhibitory effects on sebocyte proliferation through the inhibition of Akt. Linoleic acid (LA) is a well-established lipid inducer in sebocytes and
\end{abstract}

Correspondence to: Professor Beom Joon Kim, Department of Dermatology, Chung-Ang University College of Medicine, 84 Heukseok-ro, Dongjak-gu, Seoul 156-756, Republic of Korea E-mail: beomjoon@unitel.co.kr

Abbreviations: LA, linoleic acid; ERK, extracellular signalregulated protein kinase; p21, p21 ${ }^{\mathrm{WAF} 1 / \mathrm{CIP} 1} ; \mathrm{p} 27, \mathrm{p} 27^{\mathrm{KIP} 1}$; PI3-K, phosphatidylinositol 3-kinase; PPAR, peroxisome proliferator-activated receptor

Key words: resveratrol, proliferation, Akt, SZ95 human sebocytes is known to stimulate sebocyte differentiation through the upregulation of PPAR- $\gamma$. In this study, resveratrol was found to decrease the lipid content and PPAR- $\gamma$ expression during LA-stimulated lipogenesis. Our results indicate that resveratrol plays a critical role in the inhibition of sebocyte growth through the inactivation of the Akt pathway. The present data suggest that resveratrol may be used as a therapeutic agent for the treatment of acne vulgaris.

\section{Introduction}

Human skin is composed of the epidermis, dermis, hair, hair follicles and sebaceous glands. These multicellular structures consisting of a hair follicle and sebaceous glands are known as a pilosebaceous unit. In the pilosebaceous unit, the sebaceous glands store sebum, a lipid produced by sebocytes found in the skin of mammals $(1,2)$. Human sebocytes are specialized epithelial cells that secrete sebum through an accumulation of neutral lipid droplets within the cytoplasm and thus undergo terminal differentiation into adipocytes (3). Sebaceous lipogenesis results in the secretion of sebum that contains triglycerides, wax esters, squalene, cholesterol esters, cholesterol and free fatty acids. Thus, these characteristics of sebocytes are related to the functional persistence of skin by controlling the moisture balance and protecting the skin from harmful factors, such as pathogens or ultraviolet (UV) light (4-6). On the contrary, the production of excessive sebum is associated with a common inflammatory disorder of the pilosebaceous units which, along with microbial infection, is known as acne vulgaris. The pathogenesis of acne vulgaris involves a variety of factors, including increased sebum production, follicular hyperkeratinization and the induction of inflammation by Propionibacterium acnes (P. acnes) (7). Current treatments for acne vulgaris utilize topical retinoids, benzoyl peroxide, antibiotics and systemic isotretinoin (13-cis retinoic acid). It is the potent inhibition of sebum production that results in a marked reduction in the size of and the secretion of sebum by the sebaceous glands (8-10).

The growth and differentiation of sebocytes and sebum secretion are regulated by diverse factors, as well as by peroxisome 
proliferator-activated receptor (PPAR), liver X receptor (LXR) and insulin-like growth factor (IGF)-1. PPAR isoforms $(\alpha, \beta / \sigma$, $\gamma)$ are ligand-activated transcription factors found in the skin and other organs that regulate adipogenesis and lipid metabolism through cell proliferation and differentiation. In addition, PPAR isoforms are all expressed in the human sebaceous glands where lipid synthesis occurs. Furthermore, PPAR activators, such as BRL-49653, WY-14643 and linoleic acid (LA) are potent lipid inducers that result in the production of lipids through sebocyte differentiation (11-13). Studies have indicated that IGF-1 increases sebocyte proliferation and promotes sebaceous gland lipogenesis by enhancing the expression of the sterol response element binding protein-1 (SREBP-1), a transcription factor regulating lipid formation $(14,15)$. Furthermore, it has been demonstrated that IGF-1 is associated with the activation of the mitogen-activated protein-kinase/extracellular signal-regulated protein kinase (MAPK/ERK) pathway and the stimulation of the phosphatidylinositol 3-kinase (PI3-K)/Akt pathway in several cell types $(16,17)$. ERK and Akt are serine/threonine protein kinases that play a critical role in cell growth and cell survival in various cell types $(18,19)$. Taken together, both signaling pathways are known to be important for cell growth. Moreover, these pathways have been implicated in the activation of sebocytes $(16,20)$. Thus, in this study, we investigated the potential involvement of ERK and Akt in the effects exerted by treatment with resveratrol.

Resveratrol (trans-3,4',5-trihydroxystilbene) is a naturally occurring polyphenolic compound and a phytoalexin present in food products, red wine, grape skin and various plants and is produced as a reaction to environmental stresses, such as infection by plant pathogens (21). Previous studies have suggested that resveratrol exerts a variety of pharmacological effects, including antiproliferative, antioxidant, anti-inflammatory, anti-angiogenic and antimetastatic effects in many different cell lines (21-23). It has been reported that resveratrol has chemotherapeutic properties in several cell culture models and in an in vivo cancer model $(22,24)$. Previous studies have reported the inhibitory effects of resveratrol on cell cycle progression in tumor cells, which results in a reduction in the number of cells in the $\mathrm{G}_{0} / \mathrm{G}_{1}$ phase of the cell cycle and the downregulation of cyclin D1 and cyclin-dependent kinase (CDK)4 (24-27). In addition, it has been shown that resveratrol downregulates the phosphorylation of Akt and ERK in cancer cells (28). Moreover, previous studies have reported that resveratrol inhibits the proliferation of normal cells, such as smooth muscle cells and normal human keratinocytes $(29,30)$. It has also been shown to reduce the synthesis of lipids by repressing PPAR- $\gamma$ in differentiated adipocytes (31-33). Despite our understanding of the antiproliferative and pro-apoptotic properties of resveratrol in other cells, the antiproliferative signaling mechanisms of resveratrol in human sebocytes remain uncertain. Thus, in this study, in order to investigate the association between the antiproliferative effects of resveratrol and cell cycle progression, we evaluated the epxression of $\mathrm{p} 21^{\mathrm{WAF} / \mathrm{CIP1}}(\mathrm{p} 21), \mathrm{p} 27^{\mathrm{KIP1}}(\mathrm{p} 27)$ and cyclin D1 in human sebocytes.

The aim of the present study was to investigate the underlying molecular signaling pathways through which resveratrol inhibits the proliferation of human immortalized sebocytes and thus attenuates cell cycle progression. Due to the importance of cell cycle regulators in proliferation, we determined whether the levels of these regulators are affected by resveratrol. Therefore, we assessed the effects of resveratrol on cell cycle regulators in human sebocytes, and investigated the effects of this potent inhibitor of sebum production in a model of lipid droplet formation.

\section{Materials and methods}

Materials. Antibodies against phosphorylated ERK (p-ERKl; Thr202/Tyr204, \#9101S), ERK (\#9102), phosphorylated Akt (p-Akt; Ser473, \#9271S), Akt (\#9272), cyclin D1 (\#2922), p21 (\#2946) and p27 (\#3688) were purchased from Cell Signaling Technology, Inc. (Beverly, MA, USA). The antibodies to PPAR- $\gamma$ (B-5, \#sc-271392) and $\beta$-actin (I-19, \#sc-1616) were obtained from Santa Cruz Biotechnology, Inc. (Santa Cruz, CA, USA). Reagents including, LA, formaldehyde, Thiazolyl Blue tetrazolium bromide (MTT), Tris- $\mathrm{HCl}$, sodium dodecyl sulfate (SDS), $\beta$-mercaptoethanol, phenylmethylsulfonyl fluoride (PMSF), sodium orthovanadate $\left(\mathrm{Na}_{3} \mathrm{VO}_{4}\right)$, sodium fluoride $(\mathrm{NaF})$, ethylenediaminetetraacetic acid (EDTA), Oil Red O and Gill's hematoxylin were purchased from Sigma-Aldrich Co. (St. Louis, MO, USA). LY294002 (19-142, a PI3-K inhibitor) and PD98059 (19-143, a MEK inhibitor) were obtained from Calbiochem (San Diego, CA, USA). LA was dissolved in ethanol and stored at $-20^{\circ} \mathrm{C}$. Further dilutions were made in a vehicle solution (ethanol) prior to use.

Cell culture. Immortalized human sebocytes, SZ95 (34), were maintained in Sebomed ${ }^{\circledR}$ basal medium (Biochrom $\mathrm{GmbH}$, Berlin, Germany) containing 10\% fetal bovine serum (HyClone Inc., Logan, UT, USA), $1 \%$ penicillin $(10,000 \mathrm{U} / \mathrm{ml})$ and $1 \%$ streptomycin $(10,000 \mathrm{~g} / \mathrm{ml})($ Gibco-BRL, Grand Island, $\mathrm{NY}$, USA), $1 \mathrm{mM} \mathrm{CaCl}_{2}$ and $5 \mathrm{ng} / \mathrm{ml}$ human epidermal growth factor (EGF; Sigma-Aldrich Co.). The cultures were maintained at $37^{\circ} \mathrm{C}$ in a humidified $5 \% \mathrm{CO}_{2}$ incubator and the medium was replaced every 2-3 days. The SZ95 human sebocytes used for the experiments were between passages 31 and 35 .

Determination of cell proliferation by MTT assay and microscopy. The cells were examined under a phase contrast microscope (Olympus Optical Co., Tokyo, Japan) and photographed using a DCM300 digital camera (ScopeTek, Inc., Hangzhou, China), which was supported by Scope Photo software (Scopetek, Inc.). Cell proliferation was determined using the MTT reduction assay. To determine proliferation, SZ95 human sebocytes were seeded $\left(1 \times 10^{4}\right.$ cells/well) on 24-well culture plates for $24 \mathrm{~h}$ and incubated with resveratrol-containing medium at $37^{\circ} \mathrm{C}$ in $5 \% \mathrm{CO}_{2}$. Treatment with increasing concentrations of resveratrol was carried out for 24,48 and $72 \mathrm{~h}$. At the end of the resveratrol incubation period, the cells were incubated with the test substances for the indicated periods of time at $37^{\circ} \mathrm{C}$ in $5 \% \mathrm{CO}_{2}$. Subsequently, $100 \mu \mathrm{l}$ of MTT at $5 \mathrm{mg} / \mathrm{ml}$ were added to each well, and incubation was continued for $4 \mathrm{~h}$. Supernatants were removed and formazan crystals resulting from mitochondrial enzymatic activity on the MTT substrate were solubilized with dimethylsulfoxide (DMSO; Sigma-Aldrich Co.). The absorbance was measured at $540 \mathrm{~nm}$ using an enzyme-linked immunosorbent assay (ELISA) reader (VersaMax; Molecular Devices, Sunnyvale, CA, USA). 
Oil Red $O$ staining. The human SZ95 sebocytes were seeded $\left(1 \times 10^{5}\right.$ cells/well) in 6-well culture plates and incubated for $24 \mathrm{~h}$. To induce the differentiation of the sebocytes, $100 \mu \mathrm{M}$ LA were added to the culture medium and the cells were then treated with $10 \mu \mathrm{g} / \mathrm{ml}$ resveratrol for $48 \mathrm{~h}$. The cells treated with the vehicle (ethanol) served as the controls. At the end of the treatment period, the cells were washed with phosphate-buffered saline (PBS; WelGene Inc., Daegu, Korea) and fixed in $4 \%$ paraformaldehyde for $5 \mathrm{~min}$ at room temperature. After $5 \mathrm{~min}$, the cells were washed with PBS, and the fixed cells were then stained with Oil Red O solution, a mixture of Oil Red $\mathrm{O}\left(1 \%\right.$ in isopropanol) and $\mathrm{dH}_{2} \mathrm{O}$ at a ratio of $6: 4(\mathrm{vol} / \mathrm{vol})$, for $15 \mathrm{~min}$, and washed with PBS. The cell nuclei were stained with Gill's haematoxylin for $5 \mathrm{~min}$, as previously described in the study by Zouboulis et al (34). The stained cells were visualized under a microscope. Cell morphology was observed under a DP70 microscope using DP Controller software (Olympus Optical Co.). The Oil Red O staining of the lipid droplets in the cells was quantified by elution into isopropanol, and $200 \mu 1$ aliquots of the elution were then placed in 96-well plates, and the optical density of each well was determined using an ELISA reader (VersaMax; Molecular Devices) at $500 \mathrm{~nm}$. In some wells, the concentration of cellular protein from these cells was measured using a protein assay kit from Bio-Rad Laboratories, Inc. (Hercules, CA, USA). The lipid content was expressed as the percentage of living cells relative to the controls.

Determination of cell cycle changes. Following treatment of the SZ95 cells with resveratrol $(0-50 \mu \mathrm{g} / \mathrm{ml})$ for $24 \mathrm{~h}$, culture supernatants were collected to remove floating dying and apoptotic cells. The adherent cells were then harvested by trypsinization and then adjusted to $1 \times 10^{6}$ cells/tube, washed with ice-cold PBS and resuspended in $2 \mathrm{ml}$ ethanol. Following incubation at $4^{\circ} \mathrm{C}$ for $1 \mathrm{~h}$, the ethanol was removed and $100 \mu \mathrm{l}$ of ribonuclease solution $(10 \mathrm{mg} / \mathrm{ml}$, Sigma-Aldrich, Co.) were added to each tube. The tubes were then incubated at room temperature for $30 \mathrm{~min}$ and $500 \mu \mathrm{l}$ of analysis solution $(37 \mathrm{mM}$ EDTA and $0.1 \%$ Triton X-100 in PBS) and $100 \mu \mathrm{l}$ of propidium iodide (PI) solution ( $1 \mathrm{mg} / \mathrm{ml}$, Sigma-Aldrich Co.) were added. The samples were stored in the dark at $4^{\circ} \mathrm{C}$ and analyzed using a flow cytometer (FACSCalibur; Becton-Dickson, San Jose, CA, USA). The population of the cells in the sub $-\mathrm{G}_{0} / \mathrm{G}_{1}$ phase with a low DNA content was considered to be apoptotic cells.

Western blot analysis. Sebocytes were prepared in cell lysis buffer contains $62.5 \mathrm{mM}$ Tris- $\mathrm{HCl}(\mathrm{pH} 6.8), 2 \%$ SDS, $5 \% \beta$-mercaptoethanol, $2 \mathrm{mM}$ PMSF, $1 \mathrm{mM} \mathrm{Na} \mathrm{VO}_{4}$, $50 \mathrm{mM} \mathrm{NaF}$ and $10 \mathrm{mM}$ EDTA, and protease inhibitors (Roche Diagnostics, Indianapolis, IN, USA). SDS-polyacrylamide gel electrophoresis was performed using $10 \mu \mathrm{g}$ of protein per lane. The gels were blotted onto polyvinylidene fluoride (PVDF; Millipore Corp., Billerica, MA, USA) membranes that were then saturated with 5\% dried milk in Tris-buffered saline (TBS) with $0.5 \%$ Tween-20 (Sigma-Aldrich, Co.). The blots were incubated with the specific primary antibodies against p-ERK, ERK, p-AKT, AKT, PPAR- $\gamma$, cyclin D1, p21,p27 and $\beta$-actin overnight at $4{ }^{\circ} \mathrm{C}$. After washing, secondary antibodies (Cell Signaling Technology, Inc.) conjugated with horseradish peroxidase were used. Bound antibodies were detected using an ECL ${ }^{\mathrm{TM}}$ Prime Western Blotting Detection substrate (Amersham Pierce Biotechnology Inc., Rockford, IL, USA).

Statistical analysis. One-way ANOVA followed by Dunnett's T3 test were used to assess statistical significance with thresholds of $\mathrm{P}<0.05, \mathrm{P}<0.01$ and $\mathrm{P}<0.001$ indicating significant and highly significant differences, respectively.

\section{Results}

Resveratrol inhibits the proliferation of human SZ95 sebocytes. To investigate the effects of resveratrol on the proliferation of human sebocytes, we used a human SZ95 sebocyte cell line which was established by the simian virus-40 transformation of primary sebocytes derived from a human sebaceous gland (34). In the present study, in order to determine whether resveratrol exerts an antiproliferative effect on sebocytes, we treated the cells with various concentrations of resveratrol by adding it to the culture medium. Cell proliferation was assessed by MTT assay, and we found that resveratrol significantly decreased the proliferation of the SZ95 sebocytes in a dose- and time-dependent manner. Whereas cell proliferation was unaffected in the SZ95 sebocytes treated with resveratrol for $24 \mathrm{~h}$, a significant inhibitory effect of resveratrol was observed at the indicated concentration after $72 \mathrm{~h}$ of incubation (Fig. 1A). After $72 \mathrm{~h}$, treatment with resveratrol at concentrations of $1,5,10,25$ and $50 \mu \mathrm{g} / \mathrm{ml}$ decreased the cell numbers by $2.4 \pm 3.54,22.3 \pm 7.47$, $33.24 \pm 3.47,72.53 \pm 8$ and $66.46 \pm 3.49 \%$, respectively, compared to the control levels. As shown in Fig. 1B, the cell numbers were distinctly reduced following treatment with resveratrol in a dose-dependent manner. This observation indicates that resveratrol exerts antiproliferative effects on SZ95 sebocytes.

Resveratrol induces the inactivation of ERK and Akt in human SZ95 sebocytes. We demonstrated that resveratrol inhibited the proliferation of SZ95 sebocytes. In order to determine the signaling pathways through which resveratrol inhibits sebocyte proliferation, we measured the changes in ERK and/or Akt protein levels following treatment with various concentrations of resveratrol. Our results revealed that the activation of ERK was decreased by treatment of the SZ95 sebocytes with resveratrol, and that the activation of Akt was markedly inhibited in the resveratrol-treated SZ95 sebocytes (Fig. 2A). Additionally, we analyzed cell cycle distribution using DNA flow cytometry following treatment with resveratrol for $72 \mathrm{~h}$. Under the control conditions, the proportion of cells in the $G_{0} / G_{1}$ phase remained constant during the 72-h incubation period. On the contrary, the proportion of cells in the $\mathrm{G}_{0} / \mathrm{G}_{1}$ phase in the cells incubated with resveratrol was altered, and this decline was observed following treatment with $1-25 \mu \mathrm{g} / \mathrm{ml}$ of resveratrol. At high concentrations, this effect was reversible. The cell cycle distribution of the cells treated with $50 \mu \mathrm{g} / \mathrm{ml}$ of resveratrol only differed slightly from the values obtained for the control cells (Fig. 2B). Our results revealed that treatment with resveratrol resulted in a significant increase in the number of cells in the sub- $\mathrm{G}_{0} / \mathrm{G}_{1}$ phase of the cell cycle. Furthermore, we evaluated the effects of resveratrol on cell cycle progression by measuring the protein expression levels of cyclin D1, $\mathrm{p} 21^{\mathrm{WAF} 1 / \mathrm{CIP} 1}$ (p21) and $\mathrm{p} 27^{\mathrm{KIP} 1}$ (p27). As shown in Fig. 2A, 


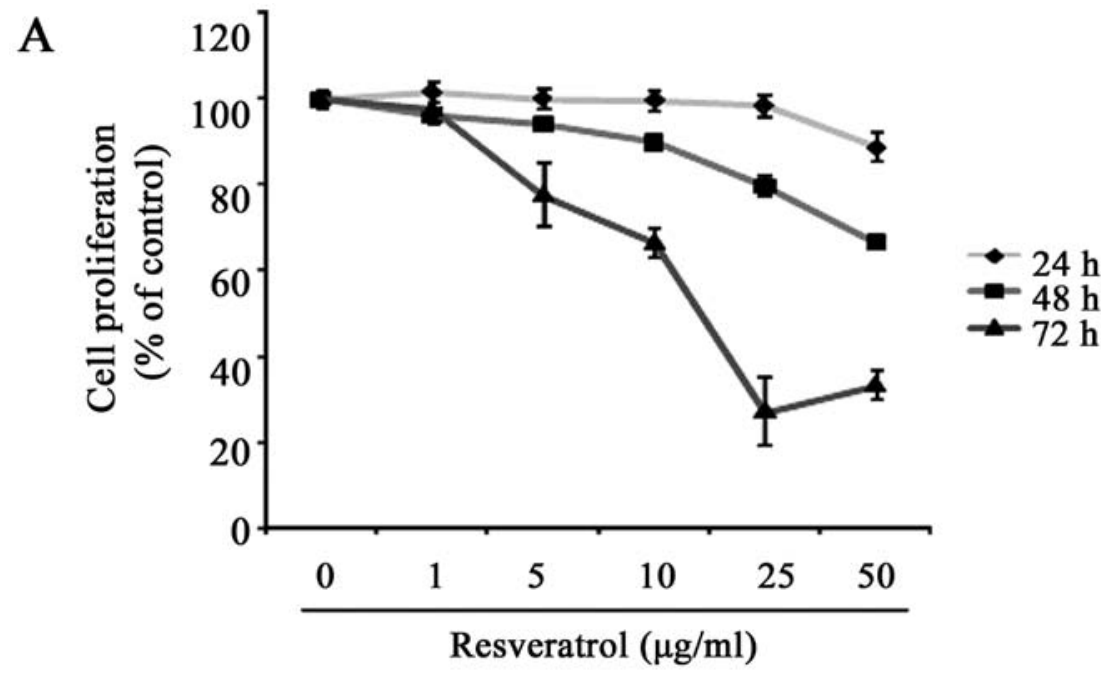

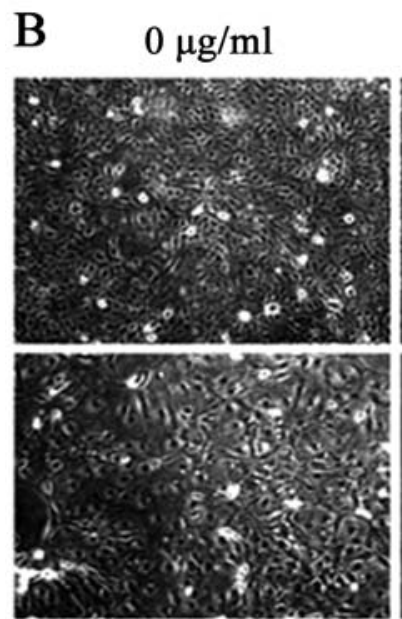

$10 \mu \mathrm{g} / \mathrm{ml}$

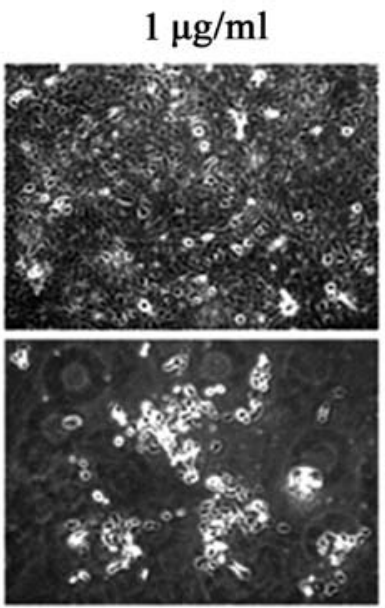

$25 \mu \mathrm{g} / \mathrm{ml}$

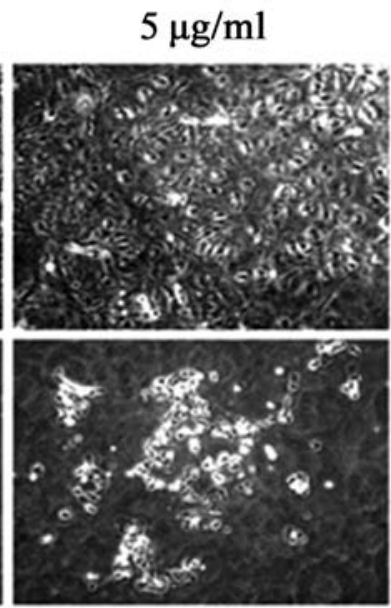

$50 \mu \mathrm{g} / \mathrm{ml}$

Figure 1. Effects of resveratrol on cell proliferation in a time-course experiment. (A) Cells were treated with various concentrations of resveratrol (1-50 $\mu \mathrm{g} / \mathrm{ml})$ for the indicated periods of time. Cell proliferation was analyzed by MTT reduction assay following treatment. Data represent the means \pm SD of triplicate assays expressed as percentages of the control. ${ }^{*} \mathrm{P}<0.05,{ }^{* * *} \mathrm{P}<0.01,{ }^{* * *} \mathrm{P}<0.001$ compared to the control. (B) Cell morphology of SZ95 sebocytes following treatment with resveratrol for $72 \mathrm{~h}$. Phase contrast photomicrographs were acquired using a DCM300 digital camera, as described in the Materials and Methods. Original magnification, $x 100$. Data are representative of 3 independent experiments.

the protein level of cyclin D1 decreased in a dose-dependent manner following treatment with resveratrol, whereas the levels of p21 and p27 increased following treatment with resveratrol. These results suggest that the resveratrol-mediated cell cycle arrest is associated with the inactivation of the ERK and Akt signaling pathways.

Resveratrol enhances the antiproliferative effects exerted by PD098059 or LY294002 in human SZ95 sebocytes. The resveratrol-treated sebocytes were cultured for $72 \mathrm{~h}$ in the presence of PD098059 (a MEK inhibitor) or LY294002 (a PI3-K inhibitor), and cell proliferation was measured by MTT assay. As shown in Fig. 3A, at a concentration $10 \mu \mathrm{g} / \mathrm{ml}$, resveratrol induced a $33 \pm 3.4 \%$ decrease in cell growth compared with the untreated controls. In order to determine the association of the ERK and Akt signaling pathways in the resveratrol-induced growth inhibitory effects, we treated the cells with the specific inhibitors, PD098059 and LY294002, and examined the antiproliferative effects of resveratrol and the inhibitors either alone or in combination. The SZ95 sebocytes were pre-treated with $50 \mu \mathrm{M}$ PD098059, a specific inhibitor of MEK, for $1 \mathrm{~h}$ and or with $50 \mu \mathrm{M}$ LY294002, a specific inhibitor of PI3-K, for $10 \mathrm{~min}$, and subsequently treated the cells with resveratrol. As shown in Fig. 3A, PD098059 alone inhibited sebocyte growth, and this PD098059-mediated inhibition was enhanced by resveratrol. In parallel, LY294002 alone inhibited sebocyte proliferation, and the combination treatment of LY294002 and resveratrol had a stronger inhibitory effect than the combination with PD098059. As a result, the inhibitory effects of

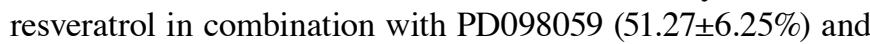
with LY294002 (77.94 $\pm 4.09 \%)$ were demonstrated (Fig. 3A). In addition, the decrease in Akt protein levels was significantly enhanced by combination treatment with LY294002 and resveratrol (Fig. 3B). These results indicate that the Akt signaling pathway is important for sebocyte proliferation.

The induction of lipogenesis by LA in human SZ95 sebocytes is blocked by resveratrol. SZ95 immortalized human 
A

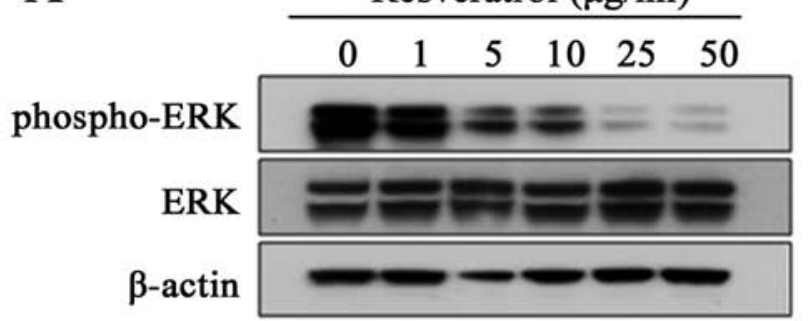

Resveratrol $(\mu \mathrm{g} / \mathrm{ml})$

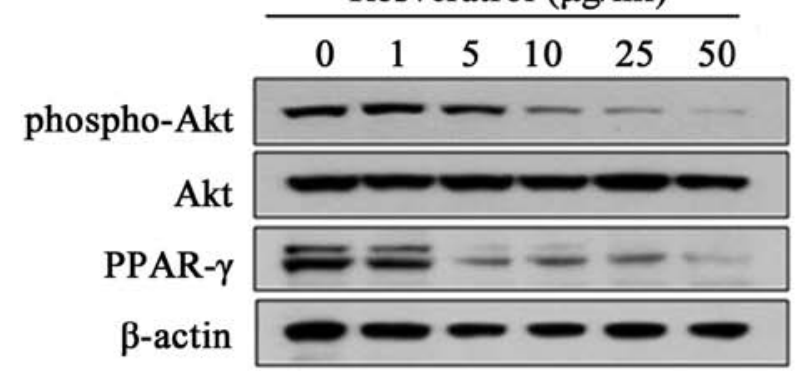

Resveratrol $(\mu \mathrm{g} / \mathrm{ml})$

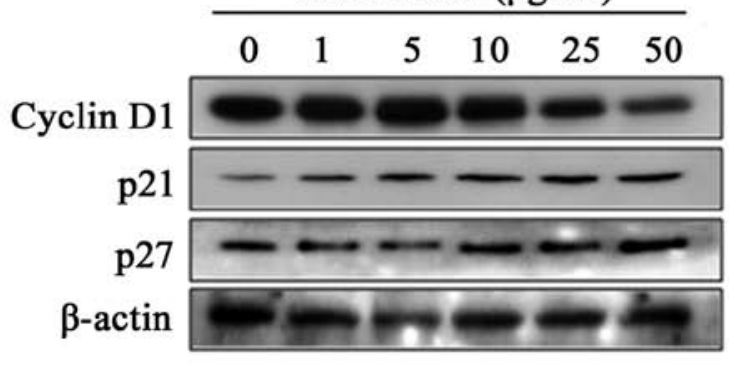

B
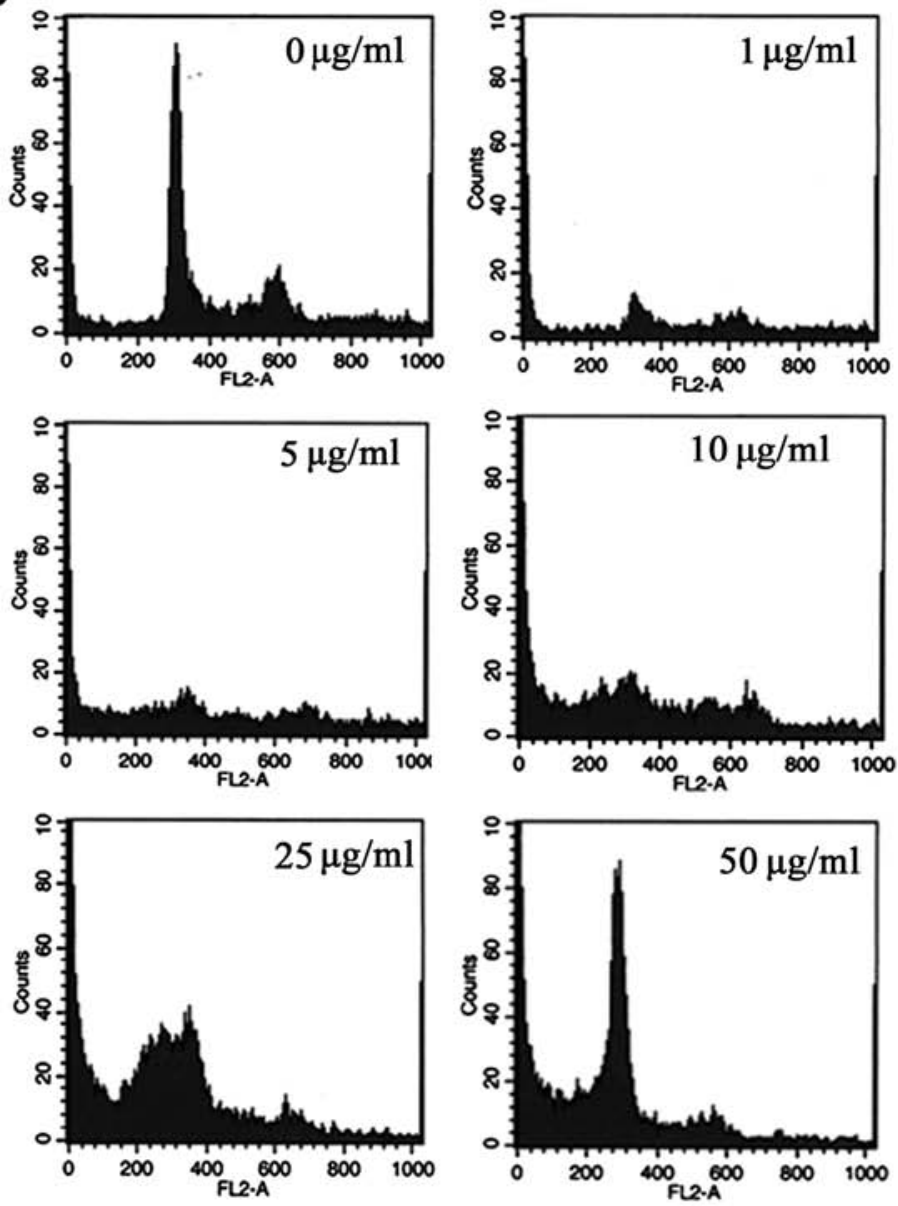

Figure 2. Effects of resveratrol on the extracellular signal-regulated protein kinase (ERK) and Akt pathways and on cell cycle control proteins in SZ95 sebocytes (A) Cells were treated with various concentrations of resveratrol $(1-50 \mu \mathrm{g} / \mathrm{ml})$ for $24 \mathrm{~h}$. Cultured cells were harvested and western blot analysis was performed with antibodies against phospho-specific ERK, phospho-specific Akt, peroxisome proliferator-activated receptor (PPAR)- $\gamma$, cyclin D1, p21 and p27. Equal protein loading was confirmed by evaluating the levels of phosphorylation-independent ERK, Akt and $\beta$-actin. (B) Cells were treated with various concentrations of resveratrol $(1-50 \mu \mathrm{g} / \mathrm{ml})$. Cell cycle analysis was performed using a flow cytometric assay, as described in the Materials and Methods. For each protein, a representative immunoblot is shown $(\mathrm{n}=3)$.

sebocyte differentiation is characterized by the intracellular accumulation of cytoplasmic lipid droplets, including neutral lipids (3). Lipid synthesis in SZ95 sebocytes is increased by diverse stimuli, such as androgens and fatty acids $(35,36)$. LA and arachidonic acid are a known as free fatty acids and induce the accumulation of lipids in SZ95 sebocytes, which activates PPAR $(37,38)$. In addition, the treatment of human sebocytes with LA $(100 \mu \mathrm{M})$ has been shown to lead to the activation of all PPAR subtypes (12). In this study, to determine the role of resveratrol during sebocyte differentiation, we examined the inhibition of lipid formation in SZ95 sebocytes by treatment with resveratrol in the presence of LA. The sebocyte lipid content was detected by Oil Red O staining and then by ELISA, and cell morphology was determined by microscopic examination, as described in the Materials and Methods. When the SZ95 sebocytes were treated with $100 \mu \mathrm{M}$ LA, Oil Red O staining revealed intracellular lipids and an enlarged number of lipid droplets in the cytoplasm surrounding the nucleus. Of note, although the intracellular lipid accumulation was further decreased in the SZ95 sebocytes following treatment with resveratrol and LA in comparison to treatment with LA alone (Fig. 4B), it was not influenced by treatment with the vehicle or by treatment with resveratrol alone. Additionally, the enhancement of lipogenesis by LA was significantly decreased in a dose-dependent manner by treatment with both resveratrol and LA (Fig. 4A), as evidenced by the results of microscopic visualization after Oil Red O staining.

To evaluate the inhibitory effect of resveratrol on PPAR- $\gamma$ activation in LA-treated cells, we examined inactivation of PPAR- $\gamma$ by resveratrol in the cells treated with LA. We found that the expression of PPAR- $\gamma$ decreased in a dose-dependent manner in the SZ95 sebocytes treated with resveratrol (Fig. 2A). As shown in Fig. 4B, LA enhanced PPAR- $\gamma$ expression in the SZ95 sebocytes, while treatment with resveratrol and LA inhibited the activation of PPAR- $\gamma$. Moreover, the activation of PPAR- $\gamma$ was inhibited by treatment with resveratrol alone in comparison to the untreated SZ95 sebocytes (Fig. 4B). These results provide evidence of the effectiveness of resveratrol against sebocyte differentiation. Thus, our results suggest that 


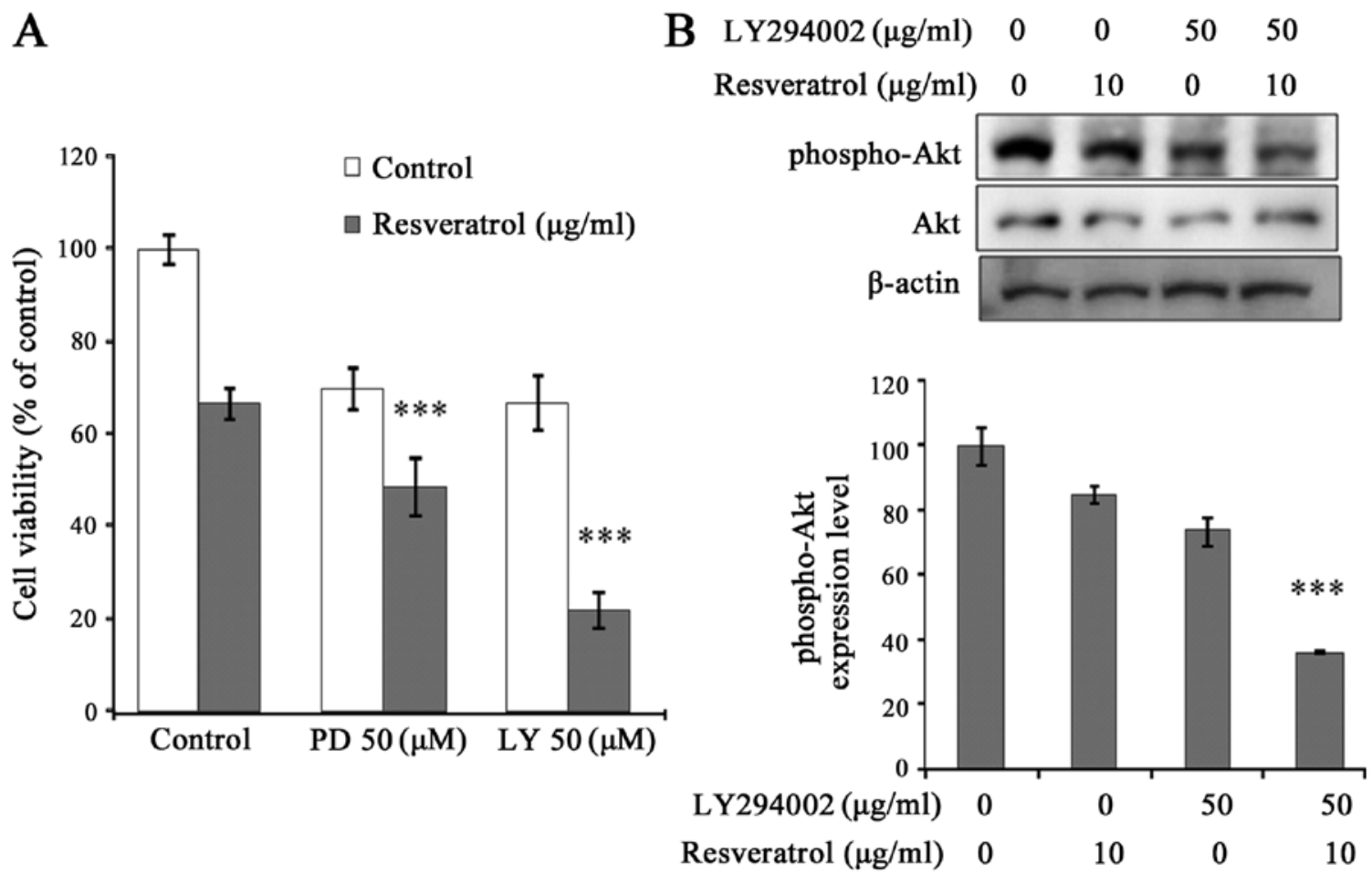

Figure 3. Effects of PD098059 (PD) and LY29002 (LY) on the inhibition of SZ95 sebocyte proliferation by resveratrol. Cells were pre-treated with $50 \mu \mathrm{M}$ PD098059 for $1 \mathrm{~h}$ or $50 \mu \mathrm{M} \mathrm{LY} 29002$ for $10 \mathrm{~min}$ alone or in combination with resveratrol $(10 \mu \mathrm{g} / \mathrm{ml})$ for $72 \mathrm{~h}$. (A) Cell proliferation was measured by MTT reduction assay. Data represent the means $\pm \mathrm{SD}$ of triplicate assays expressed as percentages of the control. ${ }^{* * *} \mathrm{P}<0.001$ compared to the $10 \mu \mathrm{g} / \mathrm{ml}$ resveratrol-treated controls. (B) Cells were treated with $10 \mu \mathrm{g} / \mathrm{ml}$ resveratrol for $24 \mathrm{~h}$. Pre-treatment was for $10 \mathrm{~min}$ with $50 \mu \mathrm{M} \mathrm{LY} 29002$. Cells were harvested and subjected to western blot analysis with antibody against phospho-specific Akt. Equal protein loading was confirmed by evaluating the levels of phosphorylation-independent Akt and $\beta$-actin. These results are representative of 3 independent experiments. Values shown are the means $\pm \mathrm{SEM}$. ${ }^{{ }^{* * *} \mathrm{P}}<0.001 \mathrm{vs}$. resveratrol-treated cells.

lipid production is reduced by resveratrol and that this reducing effect by resveratrol is mediated through the inactivation of PPAR- $\gamma$ in human sebocytes.

\section{Discussion}

The effectiveness of resveratrol (trans-3,4',5-trihydroxystilbene) in the inhibition of cell growth, cell cycle arrest and apoptosis has been investigated in several human cancer cells, and has been shown to induce anticancer effects when used as a cancer therapy $(22,24)$. In addition, a few studies have reported the antiproliferative effects of resveratrol on cell growth in normal cell types, although to date, the molecular mechanisms through which these effects occur are unknown $(29,30)$. By contrast, the antioxidant effects of resveratrol on human skin cells have been shown to be mediated by protection against hydrogen peroxide through the prevention of lipid peroxidation and programmed cell death $(14,15)$. Despite this fact, the antiproliferative effects of resveratrol in human sebocytes are not yet well understood and the mechanisms leading to the resveratrol-dependent cell growth inhibition have not been fully elucidated.

Studies utilizing a variety of cells have demonstrated the involvement of cell cycle arrest as a contributor to cell growth inhibition $(24,39,40)$. Cell cycle progression is a complex biological process that includes cell growth and mitosis. This process is controlled by CDKs, CDK inhibitors and phosphorylation events (41). Therefore, the upregulation of the p21 and p27 inhibitors of CDKs has been associated with the disruption of the cell cycle and, conversely, the decreased expression of p21 has been shown to lead to increased proliferative activity (42). A number of cell types have been shown to respond to resveratrol treatment by cell cycle arrest (24-26), the accumulation of p21 $(43,44)$ and the downregulation of cyclin D1 and CDK4 (27). In this study, to examine the association between cell growth inhibition by resveratrol and cell cycle arrest, we evaluated the involvement of the cell cycle progression machinery during the inhibition of proliferation by resveratrol in sebocytes. Our results demonstrated that the inhibition of cell growth by resveratrol involved a decrease in the expression of cyclin D1 and an enhanced expression of p21 and p27, which confirms that resveratrol exerts antiproliferative effects on SZ95 sebocytes by inducing cell cycle arrest. In addition, we demonstrated that the resveratrol-mediated cell cycle arrest resulted in an increase in the proportion of cells in the sub- $\mathrm{G}_{0} / \mathrm{G}_{1}$ phase, suggesting that resveratrol leads to the abrogation of DNA synthesis.

This study provides evidence that resveratrol significantly inhibits cell proliferation in a dose-dependent manner. Cell proliferation is regulated by a complex series of events that trigger intracellular signals, which included the activation of the ERK and Akt pathways (16-19). Therefore, as further elucidation is required, we investigated the mechanisms leading to cell growth inhibition by resveratrol in SZ95 sebocytes. The ERK pathway, one of the MAPK family proteins, is known to play key roles in cellular events, such as proliferation, differentiation and apoptosis and is activated by growth factors, such as EGF and platelet-derived growth factor (PDGF) $(45,46)$. 

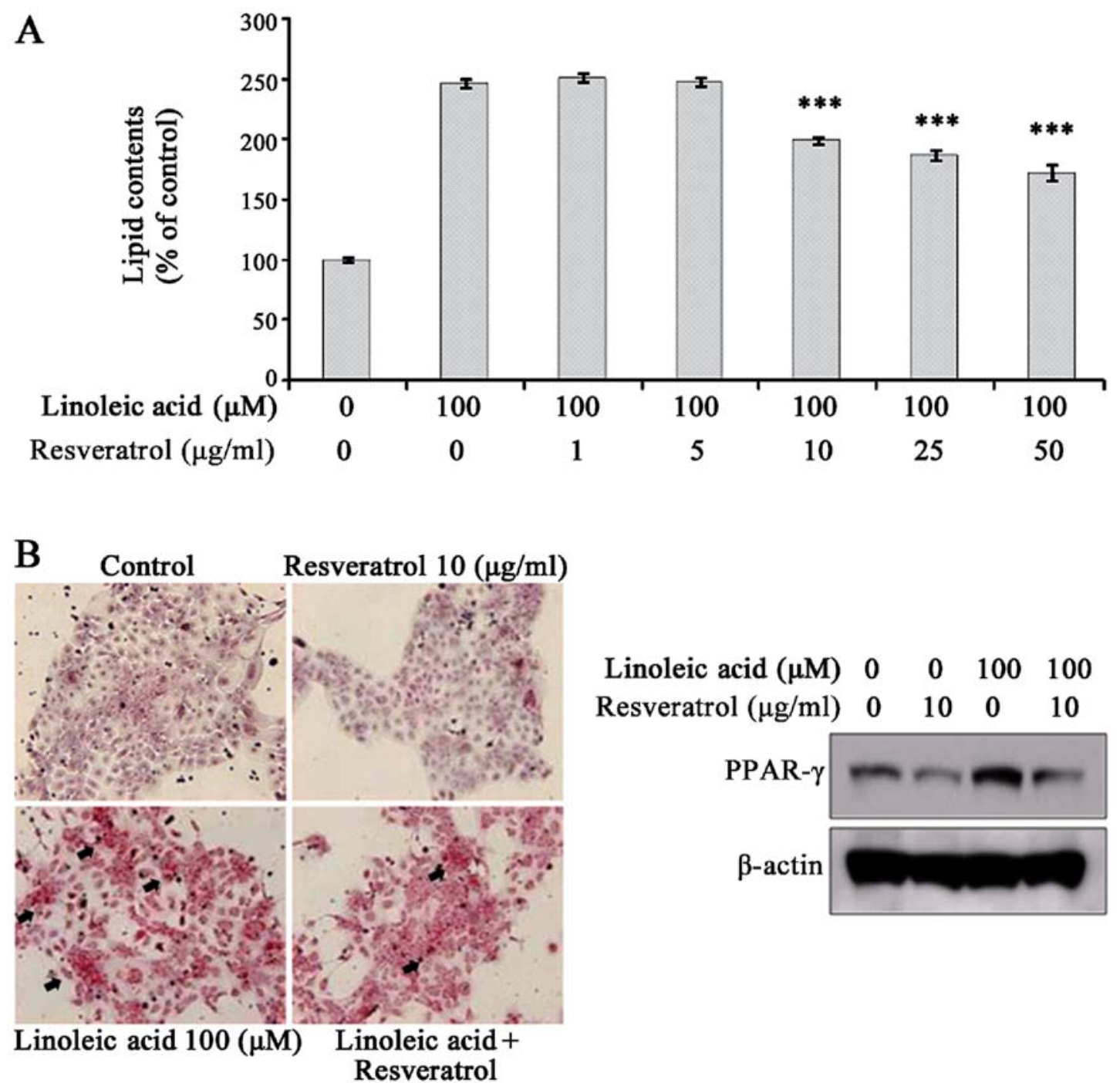

Figure 4. Effects of resveratrol on lipogenesis induced by linoleic acid (LA) and the peroxisome proliferator-activated receptor (PPAR)- $\gamma$ signaling pathway. (A) Cells were treated with various concentrations of resveratrol (1-50 $\mu \mathrm{g} / \mathrm{ml}$ ), and then incubated with linoleic acid (LA; $100 \mu \mathrm{M})$ for $48 \mathrm{~h}$. After Oil Red O staining, the lipid content was assessed by ELISA. Data represent the means $\pm \mathrm{SD}$ of triplicate assays expressed as percentages of the control. ${ }^{* * *} \mathrm{P}<0.001$ compared to the LA-treated cells. (B) Cells were treated with or without the vehicle (ethanol), $100 \mu \mathrm{M}$ linoleic acid or $10 \mu \mathrm{g} / \mathrm{ml} \mathrm{resveratrol} \mathrm{for} 48 \mathrm{~h}$. Following treatment, the lipid droplets in SZ95 sebocytes were stained with Oil Red O and visualized under a microscope. Original magnification, x200. Arrowheads indicate lipid droplets stained with Oil Red O. Cells were treated with $10 \mu \mathrm{g} / \mathrm{ml}$ resveratrol in the absence or presence of linoleic acid (100 $\mu \mathrm{M})$. Cell lysates were then subjected to western blot analysis with antibody against PPAR- $\gamma$. Equal protein loading was confirmed by evaluating the levels of $\beta$-actin. For each protein, a representative immunoblot is shown $(\mathrm{n}=3)$.

Resveratrol has previously been shown to promote growth inhibition by inhibiting the ERK pathway in K562 leukemia cells and normal human keratinocyte (47). In SZ95 sebocytes, we found that treatment with resveratrol resulted in a decrease in ERK phosphorylation in a concentration-dependent manner. In addition, our results revealed that the inhibition of the ERK pathway by PD98050 treatment, which is known to be a typical MEK inhibitor, caused cell growth inhibition, suggesting that the resveratrol-induced inactivation of ERK may be associated with an antiproliferative effect. Among the signaling pathways controlling cell growth, the Akt pathway plays a critical role in mediating both cell growth and apoptosis. Akt phosphorylation, a target signaling molecule of PI3-K, is activated when cells are exposed to IGF-1 or other growth factors (17). Our result revealed that resveratrol inactivated $\mathrm{Akt}$ in a dose-dependent manner, indicating that the antiproliferative effects of resveratrol are linked to the inactivation of the Akt pathway. Furthermore, in order to investigate the cell growth inhibition resulting from the inactivation of Akt by LY294002, a specific PI3-K inhibitor, we evaluated cell proliferation by MTT assay. Our data revealed that the inhibition of Akt by LY294002 induced cell growth inhibition, indicating that the inhibition of Akt phosphorylation by resveratrol may be involved in the growth-inhibiting mechanisms in SZ95 sebocytes.

Subsequently, we examined the effects of resveratrol on the proliferation of human sebocytes using specific inhibitors. SZ95 sebocytes were treated with resveratrol alone, or with resveratrol in combination with LY294002 or PD98050. We demonstrated that pre-treatment with PD98050 enhanced the resveratrol-mediated cell growth inhibition. In parallel, the resveratrol-induced inhibition of cell growth was increased by pre-treatment with LY294002 (Fig. 3A). Of note, the level of 
cell growth inhibition resulting from treatment with resveratrol in the presence LY294002 was greater than the antiproliferative effects of resveratrol in the presence PD098059. As shown in Fig. $3 \mathrm{~A},>67.02 \pm 4.09 \%$ of the cells were inhibited by treatment with resveratrol in the presence LY294002, compared with those treated with resveratrol only. Moreover, the phosphorylation of Akt in the resveratrol- and LY-treated cells decreased considerably to $57.23 \pm 0.26 \%$ in comparison to the cells treated with resveratrol alone (Fig. 3B). These findings suggest that resveratrol regulates cell proliferation through Akt activation, thereby suggesting a possible mechanism of action for resveratrol.

Currently, the majority of experimental and clinical studies using sebocytes are mainly focused on lipogenesis. Sebocytes are similar to adipocytes in that both cells accumulate lipids within their cytoplasms and comparable transcription factors are involved in lipid production. However, it has been reported that the secreted lipids in sebocytes differ from adipocytes-derived lipids (5). In addition, the excessive production of sebum in the sebaceous glands is pivotal in the pathogenesis of acne (7). During the process of sebum production, several transcription factors, including SREBP-1 and PPARs, are involved in lipogenic gene expression in sebocytes and are consequently involved in the pathogenesis of acne $(13,16,48)$. PPAR- $\gamma$, one of 3 PPARs subsets, has been implicated in modulating the differentiation of adipocytes and sebocytes, and its activation also enhances lipid metabolism $(11,49,50)$. In addition, it is known that the transcriptional activation of PPAR- $\gamma$ requires the activation of both the PKA and PI3-K/Akt pathways. During lipid synthesis, PI3-K/Akt signaling is an important regulatory pathway for lipogenesis in sebocytes and adipocytes, and the lack of this pathway results in a complete defect in the differentiation process $(16,51)$. Therefore, previous studies have demonstrated that resveratrol inhibits adipogenesis in 3T3-L1 adipocytes through the downregulation of PPAR- $\gamma$ and Akt signaling $(31,52)$. In addition, resveratrol has been found to inhibit lipid synthesis in rat liver and 3T3-L1 preadipocytes $(32,33)$. On the other hand, the results of a previous study investigating the effects of inhibiting lipogenesis and the mechanisms of resveratrol in sebocytes were less conclusive (53). In the present study, in order to further examine the potential anti-lipogenic effects of resveratrol, we extended our experiments to sebocyte cells. We determined the effects of resveratrol on LA-induced lipogenesis. LA is a well-established lipid inducer in sebocytes and is known to stimulate sebocyte differentiation through the upregulation of PPAR- $\gamma(11,54,55)$. In the current study, resveratrol was found to decrease the lipid content and PPAR- $\gamma$ expression in LA-stimulated lipogenesis. The treatment of the cells with resveratrol at concentrations $>5 \mu \mathrm{g} / \mathrm{ml}$ resulted in a significant reduction in their lipid content (Fig. 4A). A similar effect on cell morphology visualized by Oil Red O staining was observed using resveratrol at $10 \mu \mathrm{g} / \mathrm{ml}$, whereas resveratrol alone did not result in these changes in morphology (Fig. 4B). In addition, the effects on the expression of PPAR- $\gamma$ resulting from resveratrol treatment were investigated in human SZ95 sebocytes. Our results revealed that resveratrol resulted in a marked decrease in the expression of PPAR- $\gamma$ in a concentration-dependent manner (Fig. 2A). Moreover, as shown in Fig. 4B, resveratrol abolished the activation of LA-stimulated PPAR- $\gamma$ expression, indicating a possible mechanism of action for resveratrol. These data indicate that the potent anti-sebum effect of resveratrol is mediated through the inactivation of PPAR- $\gamma$ transcription.

In conclusion, our results demonstrated that treatment with resveratrol resulted in a dose-dependent inhibition of proliferation and induced cell cycle arrest in human SZ95 sebocytes, which was associated with the stimulation of the p21 and p27 proteins and a decrease in cyclin D1 levels. Our results demonstrated that resveratrol reduced the phosphorylation levels of ERK and Akt. LY294002 as a chemical inhibitor of the Akt pathway further enhanced the antiproliferative effects of resveratrol in contrast to the effects observed in the PD098050-treated cells, indicating that the inactivation of Akt may play a critical role in the growth inhibitory effects of resveratrol. Furthermore, resveratrol also blocked the LA-induced transcription of PPAR- $\gamma$ and sebocyte lipogenesis. These data suggest that treatment with resveratrol may be used as a therapeutic approach for the treatment of acne vulgaris.

\section{Acknowledgements}

This study was supported by a grant from the Basic Science Research Program through the National Research Foundation of Korea (NRF) funded by the Ministry of Education, Science and Technology (2010-0021960).

\section{References}

1. Zouboulis CC: Acne and sebaceous gland function. Clin Dermatol 22: 360-366, 2004.

2. Schneider MR, Schmidt-Ullrich R and Paus R: The hair follicle as a dynamic miniorgan. Curr Biol 19: R132-R142, 2009.

3. Downie MM and Kealey T: Lipogenesis in the human sebaceous gland: glycogen and glycerophosphate are substrates for the synthesis of sebum lipids. J Invest Dermatol 111: 199-205, 1998.

4. Georgel P, Crozat K, Lauth X, et al: A toll-like receptor 2-responsive lipid effector pathway protects mammals against skin infections with gram-positive bacteria. Infect Immun 73: 4512-4521, 2005.

5. Smith KR and Thiboutot DM: Thematic review series: skin lipids. Sebaceous gland lipids: friend or foe? J Lipid Res 49: 271-281, 2008.

6. Schneider MR and Paus R: Sebocytes, multifaceted epithelial cells: lipid production and holocrine secretion. Int J Biochem Cell Biol 42: 181-185, 2010.

7. Rivera AE: Acne scarring: a review and current treatment modalities. J Am Acad Dermatol 59: 659-676, 2008.

8. Goldstein JA, Socha-Szott A, Thomsen RJ, Pochi PE, Shalita AR and Strauss JS: Comparative effect of isotretinoin and etretinate on acne and sebaceous gland secretion. J Am Acad Dermatol 6 (4 Pt 2 Suppl): 760-765, 1982.

9. Zouboulis CC, Chen WC, Thornton MJ, Qin K and Rosenfield R: Sexual hormones in human skin. Horm Metab Res 39: 85-95, 2007.

10. Nelson AM, Gilliland KL, Cong Z and Thiboutot DM: 13-cis Retinoic acid induces apoptosis and cell cycle arrest in human SEB-1 sebocytes. J Invest Dermatol 126: 2178-2189, 2006.

11. Rosenfield RL, Kentsis A, Deplewski D and Ciletti N: Rat preputial sebocyte differentiation involves peroxisome proliferator-activated receptors. J Invest Dermatol 112: 226-232, 1999.

12. Deplewski D, Qin K, Ciletti N and Rosenfield RL: Unique mode of lipogenic activation in rat preputial sebocytes. J Nutr Metab 2011: 163631, 2011.

13. Rosenfield RL, Deplewski D and Greene ME: Peroxisome proliferator-activated receptors and skin development. Horm Res 54: 269-274, 2000.

14. Deplewski D and Rosenfield RL: Role of hormones in pilosebaceous unit development. Endocr Rev 21: 363-392, 2000.

15. Smith TM, Cong Z, Gilliland KL, Clawson GA and Thiboutot DM: Insulin-like growth factor-1 induces lipid production in human SEB-1 sebocytes via sterol response element-binding protein-1. J Invest Dermatol 126: 1226-1232, 2006. 
16. Smith TM, Gilliland K, Clawson GA and Thiboutot D: IGF-1 induces SREBP-1 expression and lipogenesis in SEB-1 sebocytes via activation of the phosphoinositide 3-kinase/Akt pathway. J Invest Dermatol 128: 1286-1293, 2008.

17. Decraene D, Agostinis P, Bouillon R, Degreef H and Garmyn M: Insulin-like growth factor-1-mediated AKT activation postpones the onset of ultraviolet $\mathrm{B}$-induced apoptosis, providing more time for cyclobutane thymine dimer removal in primary human keratinocytes. J Biol Chem 277: 32587-32595, 2002.

18. Blumer KJ and Johnson GL: Diversity in function and regulation of MAP kinase pathways. Trends Biochem Sci 19: 236-240, 1994

19. Franke TF, Kaplan DR and Cantley LC: PI3K: downstream AKTion blocks apoptosis. Cell 88: 435-437, 1997.

20. Choi JJ, Park MY, Lee HJ, et al: TNF- $\alpha$ increases lipogenesis via JNK and PI3K/Akt pathways in SZ95 human sebocytes. J Dermatol Sci 65: 179-188, 2012.

21. Soleas GJ, Diamandis EP and Goldberg DM: Resveratrol: a molecule whose time has come? And gone? Clin Biochem 30: 91-113, 1997.

22. Jang M, Cai L, Udeani GO, et al: Cancer chemopreventive activity of resveratrol, a natural product derived from grapes. Science 275: 218-220, 1997.

23. Clement MV, Hirpara JL, Chawdhury SH and Pervaiz S: Chemopreventive agent resveratrol, a natural product derived from grapes, triggers CD95 signaling-dependent apoptosis in human tumor cells. Blood 92: 996-1002, 1998.

24. Park JW, Choi YJ, Jang MA, et al: Chemopreventive agent resveratrol, a natural product derived from grapes, reversibly inhibits progression through S and G2 phases of the cell cycle in U937 cells. Cancer Lett 163: 43-49, 2001.

25. Hsieh TC, Juan G, Darzynkiewicz Z and Wu JM: Resveratrol increases nitric oxide synthase, induces accumulation of p53 and p21(WAF1/CIP1), and suppresses cultured bovine pulmonary artery endothelial cell proliferation by perturbing progression through S and G2. Cancer Res 59: 2596-2601, 1999.

26. Ragione FD, Cucciolla V, Borriello A, et al: Resveratrol arrests the cell division cycle at S/G2 phase transition. Biochem Biophys Res Commun 250: 53-58, 1998.

27. Wolter F, Akoglu B, Clausnitzer A and Stein J: Downregulation of the cyclin D1/Cdk4 complex occurs during resveratrol-induced cell cycle arrest in colon cancer cell lines. J Nutr 131: 2197-2203, 2001.

28. Vergara D, Simeone P, Toraldo D, et al: Resveratrol downregulates Akt/GSK and ERK signalling pathways in OVCAR-3 ovarian cancer cells. Mol Biosyst 8: 1078-1087, 2012.

29. Zou J, Huang Y, Chen Q, et al: Suppression of mitogenesis and regulation of cell cycle traverse by resveratrol in cultured smooth muscle cells. Int J Oncol 15: 647-651, 1999.

30. Holian $\mathrm{O}$ and Walter RJ: Resveratrol inhibits the proliferation of normal human keratinocytes in vitro. J Cell Biochem Suppl (Suppl) 36: 55-62, 2001

31. Rayalam S, Yang JY, Ambati S, Della-Fera MA and Baile CA: Resveratrol induces apoptosis and inhibits adipogenesis in 3T3-L1 adipocytes. Phytother Res 22: 1367-1371, 2008.

32. Arichi H, Kimura Y, Okuda H, Baba K, Kozawa M and Arichi S: Effects of stilbene components of the roots of Polygonum cuspidatum Sieb. et Zucc. on lipid metabolism. Chem Pharm Bull (Tokyo) 30: 1766-1770, 1982 .

33. Picard F, Kurtev M, Chung N, et al: Sirt1 promotes fat mobilization in white adipocytes by repressing PPAR-gamma. Nature 429: 771-776, 2004

34. Zouboulis CC, Seltmann H, Neitzel H and Orfanos CE: Establishment and characterization of an immortalized human sebaceous gland cell line (SZ95). J Invest Dermatol 113: 1011-1020, 1999.

35. Zouboulis CC and Degitz K: Androgen action on human skin-from basic research to clinical significance. Exp Dermatol 13 (Suppl 4): 5-10, 2004.

36. Zouboulis CC, Schagen S and Alestas T: The sebocyte culture: a model to study the pathophysiology of the sebaceous gland in sebostasis, seborrhoea and acne. Arch Dermatol Res 300: $397-413,2008$
37. Wróbel A, Seltmann H, Fimmel S, et al: Differentiation and apoptosis in human immortalized sebocytes. J Invest Dermatol 120: 175-181, 2003

38. Iwata C, Akimoto N, Sato T, Morokuma Y and Ito A: Augmentation of lipogenesis by 15-deoxy-Delta12, 14-prostaglandin $\mathrm{J} 2$ in hamster sebaceous glands: identification of cytochrome P-450-mediated 15-deoxy-Delta12, 14-prostaglandin J2 production. J Invest Dermatol 125: 865-872, 2005.

39. Ahmad N, Feyes DK, Nieminen AL, Agarwal R and Mukhtar H: Green tea constituent epigallocatechin-3-gallate and induction of apoptosis and cell cycle arrest in human carcinoma cells. J Natl Cancer Inst 89: 1881-1886, 1997.

40. Sugikawa E, Hosoi T, Yazaki N, Gamanuma M, Nakanishi N and Ohashi M: Mutant p53 mediated induction of cell cycle arrest and apoptosis at G1 phase by 9-hydroxyellipticine. Anticancer Res 19: 3099-3108, 1999.

41. Vermeulen K, Van Bockstaele DR and Berneman ZN: The cell cycle: a review of regulation, deregulation and therapeutic targets in cancer. Cell Prolif 36: 131-149, 2003.

42. Xiangming C, Hokita S, Natsugoe S, et al: p21 expression is a prognostic factor in patients with p53-negative gastric cancer. Cancer Lett 148: 181-188, 2000.

43. Tessitore L, Davit A, Sarotto I and Caderni G: Resveratrol depresses the growth of colorectal aberrant crypt foci by affecting bax and p21(CIP) expression. Carcinogenesis 21: 1619-1622, 2000.

44. Ahmad N, Adhami VM, Afaq F, Feyes DK and Mukhtar H: Resveratrol causes WAF-1/p21-mediated G(1)-phase arrest of cell cycle and induction of apoptosis in human epidermoid carcinoma A431 cells. Clin Cancer Res 7: 1466-1473, 2001.

45. Marshall CJ: Specificity of receptor tyrosine kinase signaling: transient versus sustained extracellular signal-regulated kinase activation. Cell 80: 179-185, 1995.

46. Karpova AY, Abe MK, Li J, et al: MEK1 is required for PDGF-induced ERK activation and DNA synthesis in tracheal myocytes. AmXJ Physiol 272: L558-L565, 1997.

47. Wu Z, Uchi H, Morino-Koga S, Shi W and Furue M: Resveratrol inhibition of human keratinocyte proliferation via SIRT1/ARNT/ERK dependent downregulation of aquaporin 3. J Dermatol Sci 75: 16-23, 2014.

48. Schuster M, Zouboulis CC, Ochsendorf F, et al: Peroxisome proliferator-activated receptor activators protect sebocytes from apoptosis: a new treatment modality for acne? Br J Dermatol 164: 182-186, 2011.

49. Rosen ED, Sarraf P, Troy AE, et al: PPAR gamma is required for the differentiation of adipose tissue in vivo and in vitro. Mol Cell 4: 611-617, 1999.

50. Rosenfield RL, Deplewski D, Kentsis A and Ciletti N: Mechanisms of androgen induction of sebocyte differentiation. Dermatology 196: 43-46, 1998.

51. Kim SP, Ha JM, Yun SJ, et al: Transcriptional activation of peroxisome proliferator-activated receptor-gamma requires activation of both protein kinase $\mathrm{A}$ and Akt during adipocyte differentiation. Biochem Biophys Res Commun 399: 55-59, 2010.

52. Floyd ZE, Wang ZQ, Kilroy G and Cefalu WT: Modulation of peroxisome proliferator-activated receptor gamma stability and transcriptional activity in adipocytes by resveratrol. Metabolism 57 (7 Suppl 1): S32-S38, 2008.

53. Docherty JJ, McEwen HA, Sweet TJ, Bailey E and Booth TD: Resveratrol inhibition of Propionibacterium acnes. J Antimicrob Chemother 59: 1182-1184, 2007.

54. McNairn AJ, Doucet Y, Demaude J, et al: TGF $\beta$ signaling regulates lipogenesis in human sebaceous glands cells. BMC Dermatol 13: 2, 2013.

55. Dozsa A, Dezso B, Toth BI, et al: PPAR $\gamma$-mediated and arachidonic acid-dependent signaling is involved in differentiation and lipid production of human sebocytes. J Invest Dermatol 134: 910-920, 2014. 